\title{
AGEÍSMO: PERCEPÇÃO DE PESSOAS IDOSAS USUÁRIAS DO CRAS
}

\section{Ageism: Perception of Elderly People Users of Cras \\ “Ageismo”: Percepción de Ancianos Usuarios del Cras \\ Âgisme : Perception des Personnes Âgées qui Utilisent le Centre de Références et d'Assistance Sociale (Cras)}

\begin{abstract}
Juliana Fernandes-Eloi 9
Doutora em Psicologia pela Universidade de Fortaleza (Unifor). Atualmente é Professora Titular da Graduação em Psicologia no Centro Universitário Estácio do Ceará, e da Graduação em Psicologia no Centro Universitário Christus.
\end{abstract}

\section{Angélica Maria de Sousa Silva 9}

Psicóloga Clínica. Mestranda em Psicologia pela Universidade de Fortaleza (Unifor).

\section{Josevânia da Silva}

Psicóloga. Doutora em Psicologia Social pela Universidade Federal da Paraíba (UFPB). Pós-Graduação em Gerontologia. Docente Adjunta do Departamento de Psicologia e Professora do Programa de Pós-graduação em Psicologia da Saúde da Universidade Estadual da Paraíba.

\section{Resumo}

O estudo teve por objetivo avaliar o preconceito contra idade vivenciado por pessoas idosas usuárias de um Centro de Referência de Assistência Social (Cras). Participaram 217 idosos, com idades entre 60 a 93 anos, sendo maioria do sexo feminino (82,9\%). Utilizou-se um questionário de caracterização sociodemográfica, a escala Ageism Survey em sua versão adaptada e um diário de campo. Os dados quantitativos foram analisados através de estatística descritiva e bivariada e os dados qualitativos, através da análise de conteúdo do tipo categorial temática. Os resultados apontaram para uma maior predominância da ocorrência de discriminação contra pessoas idosas em relação a associar dor à idade $(61,8 \%)$, ser demasiado velho para fazer algo $(57,6 \%)$ e paternalismo $(52,5 \%)$, entretanto as pessoas idosas participantes da pesquisa tendem a não perceber tais atitudes como uma forma de preconceito e/ou discriminação. É preciso maior investimento na desnaturalização da vivência do ageísmo, que se faz tão presente no cotidiano das pessoas idosas.

Palavras-chave: ageísmo; preconceito; estereótipo; velhice.

\section{Abstract}

The study aimed to assess the prejudice against age experienced by elderly users of a Social Assistance Reference Center (Cras). 217 elderly people, aged 60 to 93 years, participated, the majority being female (82.9\%). A sociodemographic characterization questionnaire, the Ageism Survey scale in its adapted version and a field diary were used. Quantitative data were analyzed using descriptive and bivariate statistics and qualitative data, through content analysis of thematic categorical type. The results showed a greater prevalence of discrimination against elderly people in relation to associating pain with age (61.8\%), being too old to do something (57.6\%) and paternalism (52.5\%), however older people participating in the survey tend not to perceive such attitudes as a form of prejudice and / or discrimination. Greater investment is needed in the denaturalization of the experience of ageism, which is so present in the daily lives of elderly people.

Keywords: ageism; preconception; stereotype; old age. 


\section{Resumen}

El estudio tuvo el objetivo de evaluar el prejuicio contra la edad vivido por ancianos usuarios de un Centro de Referencia de Atención Social (Cras). Participaron 217 ancianos, con edades entre 60 y 93 años, la mayor parte del sexo femenino (82,9\%). Fue utilizado un cuestionario de caracterización socio demográfico, la escala Ageism Survey en su versión adaptada y un diario de campo. Los datos cuantitativos fueron analizados por medio de estadística descriptiva y bivariada y los datos cualitativos, por medio de análisis de contenido del tipo categoría temática. Los resultados indicaron una mayor incidencia de discriminación contra ancianos en relación a la asociación del dolor con la edad (61,8\%), ser demasiado anciano para hacer algo (57,6\%) y paternalismo (52,5\%). Sin embargo, los ancianos participantes de la investigación tienden a no percibir tales actitudes como forma de prejuicio y/o discriminación. Es necesaria mayor inversión en la desnaturalización de la experiencia del ageismo, que es tan presente en el cotidiano de los ancianos.

Palabras clave: "ageismo"; prejuicio; estereotipo; vejez.

\section{Résumé}

L'étude a eu l'objectif à évaluer les préjugés contre l'âge subis par les personnes âgées qui utilisent un centre de référence d'assistance sociale (Cras). 217 personnes âgées (de 60 à 93 ans), ont participé de cette étude. La majorité étant des femmes (82,9\%). Un questionnaire de caractérisation sociodémographique, l'échelle "Âgisme» dans sa version adaptée et un journal de bord ont été utilisés. Les données quantitatives ont été analysées à l'aide de statistiques descriptives et bi variées et de données qualitatives, par moyen d'une analyse de contenu de type thématique catégorique. Les résultats ont montré une prévalence plus élevée de discrimination à l'égard des personnes âgées à cause d'une association de la douleur à l'âge $(61,8 \%)$; à cause d'être trop âgé pour faire n'importe quoi $(57,6 \%)$ et à cause du paternalisme (52,5\%). Pourtant, les personnes âgées qui ont participé à l'enquête ne perçoivent pas ces attitudes comme une forme de préjugés et / ou de discrimination. Il faut investir davantage dans la dénaturalisation de l'expérience de l'âgisme, si présent dans la vie quotidienne des personnes âgées.

Mots-clés : âgisme ; préjugé ; stéréotype ; vieillesse.

O aumento da expectativa de vida dos brasileiros, ainda que de modo diferenciado nas diversas regiões do país, tem colaborado para o fenômeno do envelhecimento populacional no Brasil, o que tem ocorrido em todo o mundo. Em parte, esse fenômeno está relacionado com as melhorias na qualidade de vida das pessoas, com os investimentos nas áreas da saúde, com os avanços tecnológicos, dentre outros aspectos (Camargos \& Gonzaga, 2015; Kreuz \& Franco, 2017).

Nas duas últimas décadas, devido ao aumento da expectativa de vida, tem ocorrido a ampliação de estudos e discussões sobre o envelhecimento e a velhice na realidade brasileira (Silva, Pichelli, \& Furtado, 2017; Teixeira, Marinho, Cintra Junior, \& Martins, 2015). Mais especificamente, foi a partir da década de 1960 que as ciências sociais e humanas, em parceria com a gerontologia social, intensificaram os estudos sobre os aspectos relacionados ao envelhecimento enquanto processo natural do desenvolvimento humano. Tais aspectos possuem relação com a velhice, compreendida como uma etapa do curso de vida, que é vivenciada de diversos modos e a partir dos diversos contextos e recursos de que dispõem os indivíduos (Saldanha, Silva, Furtado, \& Silva, 2019). Assim, é possível falar em velhices, em razão das pertenças sociais, das condições de vida, do acesso a bens materiais e simbólicos, entre outros aspectos.

Mesmo em face dos notáveis avanços na compreensão sobre a velhice como um fenômeno complexo e multideterminado, algumas abordagens têm por ênfase explicações meramente biológicas, caracterizando a velhice como um período de senescência, degradação fisiológica, perdas e limitações (Pereira, Freitas, \& Ferreira, 2018), o que pode colaborar para a formação de preconceitos (Torres, Camargo, \& Bousfield, 2016).

O preconceito contra idade, ou ageísmo, caracteriza-se como uma manifestação de preconceito que se fundamenta na junção de fatores de inadequação social, por motivos cronológicos, estereotipados nos modos de envelhecer, que se demarcam pela pele, roupas, hábitos sociais, econômicos e também sexuais (Palmore, 1990, 2004). Em 1969, Robert Butler utiliza o termo ageísmo pela primeira vez para designá-lo como qualquer tipo de preconceito e intolerância contra a idade, não somente ocorrido na velhice, mas durante todo o clico vital (Minichiello, Browne, \& Kendig, 2000). Posteriormente, Palmore (2004) especificou o termo ageísmo para qualquer tipo de intolerância contra os idosos de modo particular. Ademais, as investigações sobre o ageísmo têm associado o termo aos estudos sobre preconceito contra pessoas idosas (Teixeira, Souza, \& Maia, 2018).

O preconceito refere-se a "uma atitude aversiva ou hostil em relação a uma pessoa que pertence a um grupo, simplesmente por ela pertencer a esse grupo" (Allport, 1954, p.7). Na Psicologia Social, ao ser definido como uma atitude, o preconceito 
engloba um componente cognitivo (estereótipo), um componente afetivo e um componente comportamental (Álvaro \& Garrido, 2017; Teixeira et al., 2018). O componente cognitivo faz referência aos estereótipos enquanto crenças generalizadas sobre determinado grupo ou categoria social (Álvaro \& Garrido, 2017). O componente afetivo envolve as emoções positivas ou negativas relacionas ao grupo, as quais são sentidas e expressadas (Teixeira et al., 2018). Já o componente comportamental, que decorre dos elementos cognitivos e afetivos, é manifestado através das intenções e ações direcionadas ao grupo, o que caracterizaria a discriminação (Álvaro \& Garrido, 2017). A discriminação seria o comportamento ou ação hostil e injustificada direcionada a determinado grupo, bem como aos seus membros (Aronson, Wilson, \& Akert, 2015).

Pesquisas sobre preconceito contra a idade tem se ocupado em investigar suas possíveis consequências para os indivíduos e as relações interpessoais, incluindo o modo como a própria sociedade lida com o processo de envelhecimento (Castro, 2016; Silva \& França, 2015). Nessa direção, a elaboração do instrumento Ageism Survey, desenvolvido por Palmore (2004) para mensurar a discriminação contra a idade, objetivou caracterizar os três aspectos: a) a prevalência do preconceito em sociedades, b) os tipos de preconceito mais frequentes e c) os grupos que vivenciam mais preconceitos contra a idade. Concluiu-se que a vivência do ageísmo na velhice é elevada, acometendo com maior frequência os homens quando comparados com as mulheres idosas.

Na adaptação do Ageism Survey para a realidade brasileira, Couto, Koller, Novo, e Soares (2009) acrescentaram ao instrumento o item de indicadores do estresse. Os resultados indicaram que a velhice tem sido beneficiada pelos avanços que têm ocorrido na medicina e na esfera social e econômica (Couto, Koller, Novo, \& Soares, 2009), não obstante o ageísmo restringir os benefícios do prolongamento da vida para toda a sociedade. Os estereótipos relacionados às pessoas idosas são caracterizados por uma transição social que ocorre ao longo da vida (Couto \& Koller, 2012), o que demanda ações que colaborem para a desconstrução desses estereótipos em todas as etapas do desenvolvimento humano.

A partir do direcionamento de ações governamentais e políticas públicas, como o Estatuto da Pessoa Idosa, é possível construir estratégias e ações de promoção da dignidade das pessoas idosas e, consequentemente, combater preconceitos. Contudo, mesmo com a Política Nacional do Idoso e outras legislações direcionadas à proteção desse grupo etário, ainda se verifica a ocorrência de preconceito e discriminação. O que se modificou, efetivamente, foi a forma de expressão do preconceito, que, em relação aos idosos, pode ocorrer de forma sutil e benevolente (Cary, Chasteen, \& Remedios, 2017).

As pessoas tendem a não perceber o ageísmo, sendo algumas de suas práticas autorizadas socialmente, como a infantilização de idosos, os eufemismos, os paternalismos, entre outras discriminações que potencializam a vivência do sofrimento e da vulnerabilidade na velhice, bem como o isolamento e o sofrimento psíquico (Couto et al., 2009; Guerra \& Caldas, 2010). Mediante tais considerações, este estudo teve por objetivo avaliar o preconceito contra idade vivenciada por pessoas idosas usuárias de um Centro de Referência de Assistência Social (Cras).

\section{Método}

\section{Participantes}

Participaram 217 pessoas idosas usuárias de um Centro de Referência de Assistência Social - Cras, na cidade de Fortaleza - Ceará, por meio de amostra de conveniência (não probabilística), acidental e intencional. A maioria dos participantes era do sexo feminino (82,9\%), casada (36,9\%), doméstica (59\%), com idades variando de 60 a 93 anos $(\mathrm{M}=70,57 ; \mathrm{DP}=7,489)$.

\section{Instrumentos}

Aplicou-se um questionário relacionado aos aspectos sociodemográficos (idade, sexo, estado civil, entre outros), a escala Ageism Survey (Palmore, 2001, 2004 adaptado por Couto et al., 2009) e um diário de campo.

A escala Ageism Survey foi desenvolvida por Palmore $(2001,2004)$ e, posteriormente, foi adaptada e aplicada em Portugal por Alves e Novo (2006). No Brasil, foi adaptada e aplicada por Couto et al. (2009). O instrumento Ageism Survey, em sua versão adaptada para o Brasil, é composto por 20 itens, os quais abordam exemplos de estereótipos negativos, de atitudes e de discriminação pessoal e institucional contra idosos. A frequência do ocorrido é mensurada a partir de três graus de respostas: (0) - o episódio nunca ocorreu; (1) - ocorreu uma vez; e (2) - ocorreu mais do que uma vez.

Por último, um diário de campo foi utilizado para registrar as falas que emergiram dos participantes quando respondiam os itens da escala. À medida que os participantes respondiam, foi questionada a percepção que os idosos tinham sobre a vivência de tais comportamentos. O pesquisador, ao avaliar a frequência dos comportamentos contidos no instrumento, não dizia que eram vivências de discriminação para ter uma avaliação de como as pessoas idosas participantes percebiam tais expressões, se de forma positiva (isto é, não consideravam como uma atitude preconceituosa) ou negativa (isto é, era uma forma de discriminação). As respostas aos questionamentos foram anotadas e gravadas para posterior transcrição. 


\section{Procedimentos}

Esta pesquisa foi aprovada pelo comitê de ética da instituição de origem das pesquisadoras, com o CAAE: 51047215.0.0000.5038, e pela Secretaria Municipal do Trabalho, Desenvolvimento Social e Combate à Fome (Setra), responsável pela coordenação geral dos Cras de Fortaleza, que autorizou a entrada dos pesquisadores nos órgãos para a coleta dos dados. Antes da coleta de dados com os participantes, foi solicitada a assinatura do Termo de Consentimento Livre e Esclarecido (TCLE). A aplicação dos instrumentos ocorreu de forma individual, com duração aproximada de 40 minutos, no período de setembro de 2015 a março de 2016. A fim de garantir a ética e o anonimato, os relatos dos participantes foram identificados a partir de nomes de flores, bem como em razão do sexo e idade.

\section{Análise dos Dados}

Para tratamento dos dados quantitativos, foi utilizado o Statistical Package for Social Science (SPSS), versão 20. Para análise dos dados qualitativos, foi realizada a análise de conteúdo do tipo categorial temática (Bardin, 2011), que permitiu categorizar e sintetizar os sentidos, as vivências e o que emergiu a partir dos relatos dos participantes. Esse tipo de análise dá suporte na exploração dos dados, configurando-se como um método empírico e subordinado à fala/discurso. Pode-se dizer que a análise de conteúdo é um conjunto de análises de comunicação que se organiza a partir de questões abertas e nos proporciona a avaliação por categorias do conteúdo dos questionários. A análise dos dados desta pesquisa foi realizada através do levantamento de categorias temáticas, configurando-se como unidades de registro, no caso da análise de conteúdo sob um título genérico, agrupamento esse efetuado em razão dos caracteres comuns desses elementos.

\section{Resultados e Discussão}

\section{Perfil Sociodemográfico dos Participantes}

Observou-se um baixo nível de escolaridade na população estudada. A maioria dos participantes possuía o ensino fundamental incompleto, e 33,6\% dos participantes declararam não serem alfabetizados. Estes e outros dados estão demonstrados na Tabela 1.

Tabela 1.

Caracterização sociodemográfica dos idosos usuários do CRAS

\begin{tabular}{|c|c|c|}
\hline Variáveis & Frequência & $\%$ \\
\hline \multicolumn{3}{|l|}{ Sexo } \\
\hline Feminino & 789 & 82,90 \\
\hline Masculino & 428 & 37,00 \\
\hline \multicolumn{3}{|c|}{ Nível de escolaridade } \\
\hline Não alfabetizado & 52 & 33,60 \\
\hline Ensino fundamental incompleto & 03 & 41,50 \\
\hline Ensino médio & 388 & 6,90 \\
\hline Ensino superior & 180 & 3,20 \\
\hline \multicolumn{3}{|c|}{ Situação conjugal } \\
\hline Solteiros & 763 & 17,50 \\
\hline Casados & 317 & 36,90 \\
\hline Viúvos & 10 & 35,00 \\
\hline Divorciados/separados & 43 & 10,60 \\
\hline \multicolumn{3}{|l|}{ Profissão } \\
\hline Doméstica & 128 & 59,00 \\
\hline Costureira & 27 & 12,40 \\
\hline Serviços Gerais & 21 & 9,70 \\
\hline
\end{tabular}




\begin{tabular}{|c|c|c|}
\hline \multicolumn{3}{|c|}{ Prática de Atividade Física } \\
\hline Nunca & 108 & 49,80 \\
\hline Raramente & 03 & 1,40 \\
\hline Frequentemente & 103 & 47,50 \\
\hline \multicolumn{3}{|c|}{ Lazer } \\
\hline CRAS & 150 & 92,02 \\
\hline Assistir TV & 10 & 6,14 \\
\hline Visitar os filhos/netos & 03 & 1,84 \\
\hline
\end{tabular}

O nível de escolaridade das pessoas idosas participantes pode ser comparado com a pesquisa realizada pelo Instituto Brasileiro de Geografia e Estatística [IBGE], (2015), na qual foi evidenciada que 34,8\% dos idosos brasileiros possuem de quatro a oito anos de instrução, e 27,3\% dos idosos possuem menos de um ano de instrução (IBGE, 2015). Ademais, a maioria dos participantes declarou "ser doméstica" como profissão, o que tem relação com o perfil da amostra que foi, em sua maioria, composta por mulheres.

Quanto aos aspectos relacionados à saúde, embora 47,5\% dos participantes tenha afirmado realizar algum tipo de atividade física, a maioria das pessoas idosas afirmou não fazer qualquer tipo de atividade. Já em relação ao lazer, a maioria dos participantes afirmou que a vivência de atividades de lazer acorre em razão de frequentar o Cras, o que possibilita fazer diversas coisas, como dançar um "forrozinho".

O índice de pessoas idosas realizando atividade física pode ser explicado em razão da participação dessas pessoas no Cras. Uma pesquisa realizada por Brito (2018), com pessoas idosas de um Cras, evidenciou que o estilo de vida saudável esteve entre as explicações para a participação em atividade física. A manutenção da saúde foi o principal motivo para que as pessoas idosas continuassem realizando tais atividades (Brito, 2018). Enquanto política pública, além de promover ações direcionadas às atividades físicas, o Cras também tem o papel de possibilitar espaços de convivência e lazer, sobretudo para as pessoas idosas.

\section{Análise dos Dados Decorrentes do Ageism Survey}

Inicialmente, foi realizada uma análise fatorial exploratória da escala Ageism Survey. Para tanto, foi aplicando o modelo unifatorial, tendo em vista outras pesquisas já realizadas a partir desse critério (Couto et al., 2009; Palmore, 2004). Após a realização da análise fatorial da escala Ageism Survey com rotação varimax, houve a necessidade de excluir nove itens por apresentarem $\mathrm{KMO}>0,40$. A exclusão dos itens é justificada em razão da inconstância teórica presente nos dados que obedeciam ao modelo de Palmore (2004).

Os itens retirados foram os seguintes: cartão $(0,11)$; aluguel $(0,37)$; empréstimo $(0,01)$; liderança $(0,26)$; tratamento $(0,22)$; emprego $(0,20)$; promoção $(0,00)$; vandalizada $(0,05)$; violência $(0,10)$. Após a retirada desses itens, obteve-se KMO $=0,72$, teste de esfericidade de Bartlett $\operatorname{com} \chi^{2}=583,7, \mathrm{p}<0,001$ ) e alfa 0,75 fixando a extração de um fator (Tabela 2).

Quanto aos dados encontrados na administração da escala Ageism Survey, foi evidenciado que os participantes foram vítimas de ageísmo mais de uma vez. A partir dos itens assinalados pelos participantes, foram evidenciados os seguintes aspectos indicativos de ageísmo: associação de dor à idade; ser demasiado velho para realizar algo; paternalismo; assumir incompreensão; assumir surdez; ser ignorado socialmente; sofrer insulto; rejeição pela aparência; e contar anedota/piada sobre idosos. Esses resultados estão descritos na Tabela 3.

Esses resultados corroboram outros achados sobre a vivência de preconceito e discriminação que são tecidos na dinâmica das relações sociais (contar anedota; ser ignorado; sofrer insulto; paternalismo), bem como a partir de elementos baseados em estereótipos (associar dor à idade; atribuição de surdez; falta de capacidade de compreensão) (Couto et al., 2009). Tais ações caracterizam diversas formas de preconceito.

Os preconceitos são reproduzidos de forma sutil, estando embutidos nos modos de pensar e nos comportamentos relacionados aos idosos (Castro, 2016), o que tem caracterizado as novas formas de manifestação do ageísmo (Rocha, Correia, Medina, 2015; Sousa, Lodovici, Silveira, \& Arantes, 2014). O preconceito tem se apresentado através de comportamentos aceitáveis socialmente. Por exemplo, idosos com capacidade para o exercício das atividades diárias são, frequentemente, impedidos de fazê-las em razão de suposto cuidado e proteção dos parentes e pessoas próximas, contudo tais condutas podem ter relação com estereótipos sobre a velhice (Vieira \& Lima, 2015).

A vivência de situações relacionadas ao ageísmo pode gerar sofrimento psíquico, bem como outras formas de violência. Pesquisa de Silva e França (2015) realizada com 284 idosos buscou identificar os fatores que influenciam a violência contra os idosos do Rio de Janeiro. Foi aplicada a escala Ageism Survey e um questionário sociodemográfico. O estudo apontou que nível de conflito no relacionamento, dependência do idoso, sexo do idoso e renda familiar influenciam na violência contra 
Tabela 2.

Análise Fatorial da Escala de Ageism Survey

\begin{tabular}{lccc}
\hline \multicolumn{1}{c}{ Itens } & Mean & Std. Deviation & Carga Fatorial \\
\hline Incompreensão & 0,99 & 1,002 & 0,718 \\
Surdez & 0,90 & 0,998 & 0,703 \\
Aparência & 0,45 & 0,838 & 0,617 \\
Paternalismo & 1,06 & 0,998 & 0,576 \\
Dores & 1,25 & 0,964 & 0,560 \\
Velho & 1,16 & 0,988 & 0,485 \\
Respeito & 0,42 & 0,813 & 0,467 \\
Ignorado & 0,71 & 0,958 & 0,442 \\
Anedota & 0,38 & 0,785 & 0,439 \\
Insulto & 0,57 & 0,900 & 0,432 \\
Ignorado & 0,30 & 0,712 & 0,407 \\
\hline Valor próprio & & & 3,2 \\
Variância explicada & & & $29,3 \%$ \\
\hline
\end{tabular}

$\mathrm{KMO}=0,72$; Teste de esfericidade de Bartlett, $\chi^{2}=5833,6, \mathrm{p}<0,001$ ), alfa 0,75 , fixando a extração de um fator.

Tabela 3.

Frequência do Ageismo Contra Idosos

\begin{tabular}{lcc}
\hline \multicolumn{1}{c}{ Itens } & F & \% \\
\hline Associação de dores à idade & 134 & 61,8 \\
Ser demasiado velho & 125 & 57,6 \\
Paternalismo & 114 & 52,5 \\
Assumir incompreensão & 107 & 49,3 \\
Assumir surdez & 98 & 45,2 \\
Ser ignorado socialmente & 77 & 35,5 \\
Sofrer insulto & 61 & 28,1 \\
Rejeição pela aparência & 49 & 22,6 \\
Contar anedota/piada sobre idosos & 41 & 18,9 \\
\hline
\end{tabular}

eles. Os resultados evidenciaram que $44,7 \%$ dos participantes afirmou já terem sofrido algum tipo de violência. Entre os idosos que sofreram violência, a maioria $(51,1 \%)$ se caracterizava como violência psicológica, $29,9 \%$ sofreram violência mista, $12,8 \%$ violência física e 6,2\% violência financeira (Silva \& França, 2015).

$\mathrm{Na}$ contemporaneidade, as narrativas sobre a velhice ocorrem num contexto de exigências produtivas, desempenhos e metas a serem alcançadas. Sobretudo na cultura ocidental capitalista, ser velho pode significar, em geral, o fracasso diante da possibilidade existencial e produtiva do indivíduo. O ageísmo coloca a velhice sob diversos vieses estereotipados, os quais demarcam a desvalorização e inferiorização da pessoa idosa. Nesse sentido, as percepções predominantemente negativas sobre a velhice colaboram para a naturalização do ageísmo.

\section{Categorias Temáticas sobre a Vivência do Ageísmo pelas Pessoas Idosas}

Com os dados coletados através do diário de campo, a partir da análise de conteúdo categorial temática, evidenciou-se a emergência de três categorias temáticas sobre o ageísmo vivenciado pelos participantes: a) Associação de dor à idade, b) Ser extremamente velho e c) Infantilização e o paternalismo na velhice. As categorias estão descritas abaixo. 


\section{Associação de dor à idade}

De acordo com os dados encontrados na primeira categoria, que associa dores à idade, $60,8 \%$ dos participantes relataram que sua percepção acerca desse fator varia entre aceitar como uma verdade ou como algo que faz parte do processo biológico do envelhecimento. Essa concordância pode ser evidenciada nas seguintes falas: "Qualquer coisa dizem que é por causa da idade, e é mesmo"; "Mas é verdade, com a idade vai aparecendo tudo"; "Os médicos dizem é muito isso, que com a idade as doenças vão aparecendo"; "Os médicos falam é muito que essas doenças vêm da idade" (diário de campo).

Já outras pessoas idosas $(39,2 \%)$ demonstraram se incomodar com o fato de as pessoas relacionarem toda queixa de dor à idade. Esse achado pode ser evidenciado no relato de Rosa, 65 anos: "O médico veio me falar que o que eu sentia no braço era por conta da idade e eu disse que o outro braço tinha a mesma idade e estava bonzinho" (diário de campo). Portanto, verifica-se que a tentativa de se adaptar ao ageísmo, muitas vezes, é transformada em práticas sociais para aliviar os tensionamentos vividos no cotidiano.

$\mathrm{O}$ reconhecimento de que, nas últimas décadas, houve melhora significativa em termos de qualidade de vida e na expectativa de vida é evidenciado por diversos pesquisadores (Silva et al., 2017). No entanto o modo como o envelhecimento é percebido pelos indivíduos e a sociedade, embora venha passando por mudança, ainda é perpassado pela associação entre velhice e adoecimento, pela compreensão de uma etapa da vida caracterizada por problemas de saúde, destacando-se a ocorrência de dores (Brito, Camargo, \& Castro, 2017; Silva \& Fixina, 2018). Além disso, essa associação ocorre tanto entre as pessoas idosas quanto entre os profissionais de saúde.

Em relação aos aspectos físicos e de saúde, é importante destacar que o envelhecimento pode colaborar para o aumento de fragilidade e suscetibilidade, mas a velhice em si não deve ser abordada como sinônimo de doença, já que os diversos modos de envelhecer estarão relacionados com condições de vida, características do próprio indivíduo, estilo de vida, contextos de moradia, relações interpessoais, renda, escolaridade, rede de suporte social, disponibilidade de serviços em saúde, entre outros aspectos. Assim, algumas pessoas idosas poderão ter demandas em saúde e outras não. E, entre as que possuem certas demandas, há níveis diferenciados em termos de agravamento, dependência etc.

\section{Ser extremamente velho}

Em relação à categoria "ser extremamente velho", $49 \%$ dos participantes da pesquisa afirmaram já ter vivenciado comentários que associavam a idade a comportamentos que os idosos deveriam ou não deveriam mais ter. As questões do processo de envelhecimento e de reconhecimento de ser idoso sob essa nova condição existencial e de identidade estão atreladas às questões sociais, culturais e de construção ao longo da vida. Esses aspectos influenciam a forma como os idosos lidam com a velhice.

Ao serem questionados sobre se as pessoas os consideram muito velhos, $49 \%$ dos idosos afirmaram já terem ouvido considerações acerca da sua idade versus comportamentos inadequados. O relato de Orquídea, 68 anos, mostra a condição de inatividade imposta aos idosos: "Todo dia eu escuto isso, principalmente essas coisas a respeito de idade. Hoje mesmo já disseram: 'Já passou o seu tempo"” (diário de campo).

Outros participantes não se consideravam velhos, como se pode verificar no relato de Margarida, 68 anos: "Eu, velha? Todo mundo me acha nova para o que eu faço. Ninguém acredita que tenho essa idade. Sou mais nova que muito jovem" (diário de campo). Nos relatos é percebida a negação em relação à própria velhice (Guerra \& Caldas, 2010; Schneider \& Irigaray, 2008), que se subordina às exigências culturais da eterna juventude, para manterem-se jovens e ativos, em troca da aceitação social.

Embora a velhice remeta-se à um período de sabedoria e experiência, em várias situações os idosos são percebidos mais sob aspectos negativos do que positivos (Castro, 2016). Desse modo, os relatos dos participantes reforçam a naturalização, socialmente aceitável, da prática de discriminação contra os idosos (Fernandes-Eloi, Lima, \& Silva, 2019). O relato de Girassol, 72 anos, revela como a internalização do ageísmo é vivida, principalmente, entre seus parentes: "Minhas filhas são liberais, mas minhas netas, aff... Não querem que eu faça nada, eu já disse para elas que eu não morri, não" (diário de campo).

A maioria dos participantes citou que sua ida ao Cras é contra a vontade dos filhos, netos e parentes, porém os idosos sentem satisfação em estar nesse ambiente e participar do Cras. Embora os participantes tenham relatado que entendem seus filhos, o processo de retirada de autonomia da pessoa idosa, ou mesmo a superproteção, colabora para práticas que despotencializam e desacreditam a capacidade desse grupo etário, o que corrobora para o paternalismo e a infantilização dos idosos como expressão do ageísmo (Palmore, 2004).

A literatura discute que a manifestação do ageísmo pode ocorrer de modo hostil e/ou benevolente (Cary et al., 2017). O ageísmo benevolente ou positivo ocorre de maneira sutil e, aparentemente, apresentam-se de modo positivo, mas por trás revela uma fragilidade e uma retirada da autonomia dos idosos. Embora a escala Ageism Survey esteja voltada para a avaliação do ageísmo hostil, percebe-se tanto a manifestação do ageísmo hostil quanto a presença do ageísmo benevolente nas falas dos participantes. 


\section{Infantilização e o paternalismo na velhice}

A infantilização e o paternalismo foi a terceira maior vivência de discriminação que as pessoas idosas participantes da pesquisa relataram ter vivenciado $(52,5 \%)$. Esse dado revela que a postura infantilizada em relação à pessoa idosa geralmente esconde a presença de uma violência simbólica e complexa de ser percebida, apreendida e reconhecida, tanto por quem a exerce como pelo próprio idoso. O fenômeno da infantilização e da paternalização corroboram o que "os gerontólogos sociais sustentam, que as atitudes preconceituosas em relação à velhice determinam práticas sociais que contribuem para a manutenção de ideias discriminativas, preconceituosas e paternalistas" (Silva, Farias, Oliveira, \& Rabelo, 2012, p. 122).

Entretanto, quando se questionou sobre a percepção que esses idosos tinham acerca do ocorrido, foi verificado que, enquanto afirmam que as pessoas expressam um carinho exagerado (paternalismo) quando se dirigem a eles, $65,4 \%$ dos idosos não percebem essa relação como algo negativo ou preconceito. Pelo contrário, Lírio, 75 anos afirma: "É uma forma de carinho, pelo menos estão olhando pra gente" (diário de campo). Esse dado pode confirmar o que as pesquisas postulam em relação às novas formas de expressão do ageísmo, manifestado através de comportamentos aceitáveis socialmente (Fernandes-Eloi, Dias, Nunes, \& Silva, 2019), porém tais expressões estão carregadas ou escondem uma visão de fragilidade e infantilidade que possuem perante as pessoas idosas (Nelson, 2005).

Entende-se por paternalismo a realização de comportamentos de autoridade de uma pessoa sobre outra. Nesse contexto, o paternalismo se apresenta através do comportamento dos filhos que exercem as funções típicas da paternidade e passam a superproteger seus pais, ou seja, ao exercer o paternalismo, uma pessoa toma decisões que interferem no cotidiano do outro. Portanto, de acordo com os achados neste estudo, a maioria das pessoas já foi tratada de forma infantilizada, e muitas, chegaram a ser proibidas pelos filhos de frequentar o Cras.

\section{Considerações Finais}

Neste estudo pôde-se verificar tanto a frequência da vivência da discriminação como a naturalização do ageísmo. Entre os tipos de discriminação contra idosos, foram evidenciados os seguintes aspectos: a associação de dorà idade, ser demasiado velho para realizar algo e o paternalismo. As percepções negativas atreladas ao processo de envelhecimento corroboram as práticas de exclusão dos idosos.

A compreensão sobre os achados do estudo deve considerar o contexto das pessoas idosas participantes da pesquisa. Elas estavam inseridas numa realidade de política pública que representava, para a maioria dos idosos, a oportunidade para experimentarem um ambiente de lazer. Ademais, os participantes da pesquisa mantinham presença constante nas atividades do Cras, o que demonstra a importância de políticas públicas que promovam o bem-estar e a qualidade de vida das pessoas idosas.

Sugere-se que sejam construídos novos instrumentos metodológicos que avaliem o preconceito e a discriminação contra idosos levando em conta as diversas velhices existentes na realidade brasileira, como as diferenças existentes entre os contextos urbanos e rurais. Vale-se ressaltar que a escala utilizada neste estudo apresenta uma variabilidade de respostas limitadas ao fenômeno do ageísmo ( 0 - o episódio nunca ocorreu; 1 - ocorreu uma vez; e 2 - ocorreu mais do que uma vez), de modo que se faz necessário considerar uma variabilidade maior de pontuações a fim de compreender as nuances que perpassam a vivência do ageísmo.

\section{Referências}

Allport, G. W. (1954). The nature of prejudice ( $3^{\mathrm{a}}$ ed.). Wokingham: Addison-Wesley.

Álvaro, J. L., \& Garrido, A. (2017). Psicologia social: perspectivas psicológicas e sociológicas. Artmed Editora.

Alves, J. F., \& Novo, R. F. (2006). Avaliação da discriminação social de pessoas idosas em Portugal. International Journal of Clinical and Health Psychology, 6(1), 65-77.

Aronson, E., Wilson, T. D., \& Akert, R. M. (2015). Preconceito: causas e curas. In E. Aronson (Ed.), Psicologia Social (8a ed). Rio de Janeiro: Livros Técnicos e Científicos Editora.

Bardin, L. (2011). Content analysis. São Paulo: Edições, 70, 279.

Brito, A. M. M., Camargo, B. V., \& Castro, A. (2017). Representações sociais de velhice e boa velhice entre idosos e sua rede social. Revista de Psicologia da IMED, 9(1), 5-21. DOI: 10.18256/2175-5027.2017.v9i1.1416 
Brito, T. L. F. (2018). Fatores motivacionais para a prática de exercício físico em idosos. Trabalho de Conclusão de Curso (Graduação) - Universidade Federal do Pará, Castanhal, Pará. Link

Camargos, M. C. S., \& Gonzaga, M. R. (2015). Live longer and better? Estimates of healthy life expectancy in the Brazilian population. Cadernos de saúde publica, 31(7), 1460-1472. DOI: 10.1590/0102-311X00128914

Cary, L. A., Chasteen, A. L., \& Remedios, J. (2017). The ambivalent ageism scale: Developing and validating a scale to measure benevolent and hostile ageism. The Gerontologist, 57(2), e27-e36. DOI: 10.1093/geront/gnw118

Castro, G. G. S. (2016). Ageism as a cultural trend: Reflections on the production of meanings regarding longer life in today’s world. Galáxia (São Paulo), (31), 79-91. DOI: 10.1590/1982-25542016120675

Couto, M. C. P. P., \& Koller, S. H. (2012). Warmth and competence: Stereotypes of the elderly among young adults and older persons in Brazil. International Perspectives in Psychology: Research, Practice, Consultation, 1(1), 52. DOI: 10.1037/ a0027118

Couto, M. C. P. P., Koller, S. H., Novo, R., \& Soares, P. S. (2009). Avaliação de discriminação contra idosos em contexto brasileiro - ageismo. Psicologia: Teoria e Pesquisa, 25, 509-518. DOI: 10.1590/S0102-37722009000400006

Fernandes-Eloi, J., Dias, M. D. F., Nunes, T. R. T., \& Silva, A. M. S. (2019). Afetos e percepções de idosos universitários acerca do mercado de trabalho na velhice. Revista Kairós: Gerontologia, 22(1), 249-271. Link

Fernandes-Eloi, J., Lima, M. E. G., \& Silva, A. M. S. (2019). Reinserção de idosos no mercado de trabalho: uma etnografia de tela do filme Um Sr. Estagiário. Pensando familias, 23(1), 199-212. Link

Guerra, A. C. L. C., \& Caldas, C. P. (2010). Dificuldades e recompensas no processo de envelhecimento: A percepção do sujeito idoso. Ciência \& Saúde Coletiva, 15(6), 2931-2940. DOI: 10.1590/S1413-81232010000600031

Instituto Brasileiro de Geografia e Estatística. (2015). Síntese de indicadores sociais: Uma análise das condições de vida da população brasileira. Link

Kreuz, G., \& Franco, M. H. P. (2017). Reflexões acerca do envelhecimento, problemáticas, e cuidados com as pessoas idosas. Revista Kairós: Gerontologia, 20(2), 117-133. DOI: 10.23925/2176-901X.2017v20i2p117-133

Minichiello, V., Browne, J., \& Kendig, H. (2000). Perceptions and consequences of ageism: views of older people. Ageing \& Society, 20(3), 253-278. DOI: 10.1017/S0144686X99007710

Nelson, T. D. (2005). Ageism: Prejudice against our feared future self. Journal of Social Issues, 61, 207-221. DOI: 10.1111/j.1540-4560.2005.00402.x

Palmore, E. B. (1990). Ageism: Positive and negative. New York: Springer.

Palmore, E. B. (2001). The ageism survey: First findings. The Gerontologist 41, 1-3. DOI: 10.1093/geront/41.5.572

Palmore, E. B. (2004). Research note: Ageism in Canada and the United States. Journal of Cross-Cultural Gerontology, 19(1), 41-46. DOI: 10.1023/B:JCCG.0000015098.62691.ab

Pereira, R. F., Freitas, M. C., \& Ferreira, M. A. (2018). Velhice para os adolescentes: Abordagem das representações sociais. Revista Brasileira de Enfermagem, 67(4), 601-609. DOI: 10.1590/0034-7167.2014670416

Rocha, P. A. L., Correia, J.A., \& Medina, M. T. (2015). A(re)construção de contextos e culturas da velhice e do envelhecimento: Abordagens da gerontologia social/crítica. Trabalho \& Educação, 24(2), 57-70.

Saldanha, A. A. W., Silva, J., Furtado, F. M. S. F, \& Silva, V. F. (2019). Vulnerabilidades em saúde no contexto rural. In S. C. 
Maciel \& P. N. Fonsêca, Psicologia Social: Vertentes e perspectivas (pp.147-164). João Pessoa: Editora UFPB.

Schneider, R. H., \& Irigaray, T. Q. (2008). O envelhecimento na atualidade: Aspectos cronológicos, biológicos, psicológicos e sociais. Estudos de Psicologia, 25(4), 585-593. DOI: 10.1590/S0103-166X2008000400013

Silva, L. C. C., Farias, L. M. B., de Oliveira, T. S., \& Rabelo, D. F. (2012). Atitude de idosos em relação à velhice e bem-estar psicológico. Revista Kairós: Gerontologia, 15(2), 119-140.

Silva, C. A. A., \& Fixina, E. B. (2018). Significados da velhice e expectativas de futuro sob a ótica de idosos. Geriatrics, Gerontolology and Aging, 12(1), 8-14. DOI: 10.5327/Z2447-211520181700081

Silva, E. A., \& França, L. H. F. P. (2015). Violência contra idosos na cidade do Rio de Janeiro. Estudos e pesquisas em Psicologia, 15(1), 155-177. DOI: 10.12957/epp.2015.16067

Silva, J., Pichelli, A. A. W. S., \& Furtado, F. M. S. (2017). O envelhecimento em cidades rurais e a análise das vulnerabilidades em saúde. In C. M. R. G. Carvalho \& L. F. Araújo. Envelhecimento e práticas gerontológicas (pp. 291-310). Curitiba/ Teresina: CRV EDUFPI.

Sousa, A. C. S. N., Lodovici, F. M. M., Silveira, N. D. R., \& Arantes, R. P. G. (2014). Alguns apontamentos sobre o Idadismo: A posição de pessoas idosas diante desse agravo à sua subjetividade. Estudos Interdisciplinares sobre o Envelhecimento, 19(3), 853-877. Link

Teixeira, S. M. O., Marinho, F. X. S., Cintra Junior, D. F., \& Martins, J. C. O. (2015). Reflexões acerca do estigma do envelhecer na contemporaneidade. Estudos interdisciplinares sobre o envelhecimento, 20(2), 503-515. Link

Teixeira, S. M. O., Souza, L. E. C., \& Maia, L. M. (2018). Ageísmo institucionalizado: Uma revisão teórica. Revista KairósGerontologia, 21(3), 129-149. Link

Torres, T. L., Camargo, B. V., \& Bousfield, A. B. S. (2016). Estereótipos sociais do idoso para diferentes grupos etários. Psicologia: teoria e pesquisa, 32(1), 209-219. DOI: 10.1590/0102-37722016012114209218

Vieira, R. S., \& Lima, M. E. O. (2015). Estereótipos sobre os idosos: Dissociação entre crenças pessoais e coletivas. Temas em Psicologia, 23(4), 947-958. DOI: 10.9788/TP2015.4-12 


\section{Como citar:}

Fernandes-Eloi, J., Silva, A. M. S., Silva, J. (2020). Ageísmo: Percepção de Pessoas Idosas Usuárias do Cras. Revista Subjetividades, 20(Esp 1. Relações Intergrupais: Preconceito e Exclusão Social), e8945. http://doi.org/10.5020/23590777. rs.v20iEsp1.e8945

\section{Endereço para correspondência}

Juliana Fernandes-Eloi

E-mail: julianafernandeseloi@gmail.com

Angélica Maria de Sousa Silva

E-mail: angelica_psi.silva@hotmail.com

Josevânia da Silva

E-mail: josevaniasco@gmail.com 